\title{
MEROMORPHIC EXTENDIBILITY AND THE ARGUMENT PRINCIPLE
}

\author{
Josip Globevnik
}

\begin{abstract}
Let $\Delta$ be the open unit disc in $\mathbb{C}$. Given a continuous function $\varphi: b \Delta \rightarrow \mathbb{C} \backslash\{0\}$ denote by $\mathcal{W}(\varphi)$ the winding number of $\varphi$ around the origin. We prove that a continuous function $f: b \Delta \rightarrow \mathbb{C}$ extends meromorphically through $\Delta$ if and only if there is a number $N \in \mathbb{N} \cup\{0\}$ such that $\mathcal{W}(P f+Q) \geq-N$ for every pair $P, Q$ of polynomials such that $P f+Q \neq 0$ on $b \Delta$. If this is the case then the meromorphic extension has at most $N$ poles in $\Delta$.
\end{abstract}

\section{Introduction and the main result}

Let $\Delta$ be the open unit disc in $\mathbb{C}$ and let $f: b \Delta \rightarrow \mathbb{C}$ be a continuous function. We say that $f$ extends holomorphically through $\Delta$ if $f$ admits a continous extension $\tilde{f}$ to $\bar{\Delta}$ which is holomorphic on $\Delta$. If this is the case then we say that $f$ (or $\tilde{f}$ ) belongs to the disc algebra. Denote by $\overline{\mathbb{C}}=\mathbb{C} \cup\{\infty\}$ the Riemann sphere. We say that $f$ extends meromorphically through $\Delta$ if there is a finite set $A \subset \Delta$ such that $f$ has a continuous extension to $\bar{\Delta} \backslash A$ which is holomorphic on $\Delta \backslash A$ and has a pole at each point of $A$. Equivalently, $f$ extends meromorphically through $\Delta$ if it has a continous extension $\tilde{f}: \bar{\Delta} \rightarrow \overline{\mathbb{C}}$ which, as a function to $\overline{\mathbb{C}}$, is holomorphic on $\Delta$.

Given a continuous function $\varphi: b \Delta \rightarrow \mathbb{C} \backslash\{0\}$ we denote by $\mathcal{W}(\varphi)$ the winding number of $\varphi$ (around the origin). So $\mathcal{W}(\varphi)$ equals $1 /(2 \pi)$ times the change of argument of $\varphi(z)$ as $z$ runs once around $b \Delta$ counterclockwise.

In the present paper we show that meromorphic extendibility can be characterized in terms of the argument principle. For holomorphic extendibility this is already known:

THEOREM 1.0 [G2] A continuous function $f: b \Delta \rightarrow \mathbb{C}$ extends holomorphically through $b \Delta$ if and only if $\mathcal{W}(f+Q) \geq 0$ for every polynomial $Q$ such that $f+Q \neq 0$ on $b \Delta$.

If a continuous function $f: b \Delta \rightarrow \mathbb{C} \backslash\{0\}$ extends meromorphically through $\Delta$ then $\mathcal{W}(f) \geq-N$ where $N$ is the number of poles of the meromorphic extension $\tilde{f}$ (counted with multiplicity). Indeed, by the argument principle,

$$
\mathcal{W}(f)=\nu_{0}(\tilde{f})-\nu_{p}(\tilde{f})=\nu_{0}(\tilde{f})-N \geq-N
$$

where $\nu_{0}(\tilde{f})$ is the number of zeros of $\tilde{f}$ on $\Delta$ and $\nu_{p}(\tilde{f})$ is the number of poles of $\tilde{f}$ on $\Delta$.

Let $f: b \Delta \rightarrow \mathbb{C}$ be a continuous function on $b \Delta$ which extends meromorphically through $\Delta$ and whose meromorphic extension $\tilde{f}$ has $N$ poles on $\Delta$. Then $\mathcal{W}(P f+Q) \geq-N$ 
for all polynomials $P, Q$ such that $P f+Q \neq 0$ on $b \Delta$. Indeed, $P \tilde{f}+Q$, the meromorphic extension of $P f+Q$, has no other poles than $\tilde{f}$ and therefore, by the argument principle, $\mathcal{W}(P f+Q) \geq-N$. The following theorem, our main result, tells that this property characterizes meromorphic extendibility.

THEOREM 1.1 A continuous function $f: b \Delta \rightarrow \mathbb{C}$ extends meromorphically through $\Delta$ if and only if there is an $N \in \mathbb{N} \cup\{0\}$ such that

$$
\mathcal{W}(P f+Q) \geq-N
$$

for all polynomials $P, Q$ such that $P f+Q \neq 0$ on $b \Delta$. If this is the case then the meromorphic extension of $f$ has at most $N$ poles in $\Delta$, counting multiplicity.

\section{Fourier series}

In this section we recall some well known facts.

Let $\mathrm{f}$ be a continuous function on $b \Delta$. For each integer $n$ let

$$
\hat{f}(n)=\frac{1}{2 \pi} \int_{-\pi}^{\pi} e^{-i n \theta} f\left(e^{i \theta}\right) d \theta
$$

so that

$$
\sum_{n=-\infty}^{\infty} \hat{f}(n) e^{i n \theta}
$$

is the Fourier series of $f$. We have

$$
\sum_{n=-\infty}^{\infty}|\hat{f}(n)|^{2}<\infty
$$

Define the functions $F$ and $G$ by

$$
\begin{gathered}
F(z)=\hat{f}(0)+\hat{f}(1) z+\hat{f}(2) z^{2}+\cdots \quad(z \in \Delta) \\
G(z)=\overline{\hat{f}(-1)} z+\overline{\hat{f}(-2)} z^{2}+\cdots \quad(z \in \Delta) .
\end{gathered}
$$

The functions $F$ and $G$ are holomorphic on $\Delta$ and by (2.1) they belong to the space $H^{2}$ $[\mathrm{R}]$.

The function $f$ belongs to the disc algebra if and only if $\hat{f}(n)=0$ for all $n<0$ or, equivalently, if and only if $G \equiv 0$.

Suppose now that $f$ is smooth. Then the Fourier series converges uniformly to $f$. The functions $F$ and $G$ belong to the disc algebra and have smooth boundary values. We have

$$
f(z)=F(z)+\overline{G(z)}(z \in b \Delta) .
$$

We shall need the following 
PROPOSITION 2.1 Let $\Phi: b \Delta \rightarrow \mathbb{C}$ be a continuous function. Given $N \in \mathbb{N}$ there is a nonzero polynomial $P$ of degree not exceeding $N$ such that $(\hat{P \Phi})(j)=0(-N \leq j \leq-1)$.

Proof. $P \Phi$ is continuous on $\Delta$ and a direct computation shows that for each integer $j$ we have

$$
(\hat{P \Phi})(j)=\hat{P}(0) \hat{\Phi}(j)+\hat{P}(1) \hat{\Phi}(j-1)+\cdots+\hat{P}(N) \hat{\Phi}(j-N)
$$

so $(\hat{P F})(j)=0(-N \leq j \leq-1)$ gives the homogeneous system

$$
\begin{aligned}
& \hat{P}(0) \hat{\Phi}(-N)+\hat{P}(1) \hat{\Phi}(-N-1)+\cdots+\hat{P}(N) \hat{\Phi}(-2 N)=0 \\
& \hat{P}(0) \hat{\Phi}(-N+1)+\hat{P}(1) \hat{\Phi}(-N)+\cdots+\hat{P}(N) \hat{\Phi}(-2 N+1)=0 \\
& \cdots \\
& \hat{P}(0) \hat{\Phi}(-1)+\hat{P}(1) \hat{\Phi}(-2)+\cdots+\hat{P}(N) \hat{\Phi}(-N-1)=0
\end{aligned}
$$

of $N$ linear equations with $N+1$ unknowns $\hat{P}(0), \hat{P}(1), \cdots, \hat{P}(N)$ which always has a nontrivial solution. This completes the proof.

\section{The smooth case}

THEOREM 3.1 Suppose that $f: b \Delta \rightarrow \mathbb{C}$ is of the form

$$
f(z)=G(z)+\overline{H(z)} \quad(z \in b \Delta)
$$

where the functions $G$ and $H$ belong to the disc algebra and $H$ has smooth boundary values. Assume that $N \in \mathbb{N} \cup\{0\}$ and that

$$
\left.\begin{array}{r}
\mathcal{W}(P f+Q) \geq-N \text { whenever } P, Q, \text { are polynomials, } \\
\operatorname{deg}(P) \leq N, \text { such that } P f+Q \neq 0 \text { on } b \Delta .
\end{array}\right\}
$$

Then $f$ extends meromorphically through $\Delta$ and the meromorphic extension has at most $N$ poles in $\Delta$, counting multiplicity.

REMARK 3.2 To prove Theorem 1.1 we shall later use Theorem 3.1 only in the special case when $H$ is a rational function holomorphic in a neighbourhood of $\bar{\Delta}$ so with this in mind, we may assume as much smoothness as we want. In the proof of Theorem 3.1 below it is enough to assume that $H \mid b \Delta$ belongs to the Lipschitz class $C^{\alpha}$ with $\alpha>1 / 2$.

Before proceeding observe that if $f$ is as in Theorem 3.1 and $P$ is a polynomial then $P f$ has the same form. Indeed, we have $P f=P G+P \bar{H}$ on $b \Delta$ where the function $z \mapsto$ $P(z) \overline{H(z)}$ is smooth on $b \Delta$ so on $b \Delta$ we have $P \bar{H}=F_{2}+\overline{G_{1}}$ where $F_{2}, G_{1}$ belong to the disc algebra and have smooth boundary values. So on $b \Delta$ we have $P f=P G+F_{2}+\overline{G_{1}}=F_{1}+\overline{G_{1}}$ where $F_{1}, G_{1}$ are in the disc algebra and $G_{1}$ has smooth boundary values.

Proof of Theorem 3.1. Assume that $f$ is as in Theorem 3.1 and that (3.1) holds for some $N \in \mathbb{N} \cup\{0\}$. If $N=0$ then it is known that $f$ extends holomorphically through $\Delta$ [G2]. Assume that $N \geq 1$. By Proposition 2.1 there is a polynomial $P, \operatorname{deg}(P) \leq N$, such that

$$
\hat{P f})(-1)=(\hat{P f})(-2)=\cdots=(\hat{P f})(-N)=0
$$


Now, $P f=F_{1}+\overline{G_{1}}$ on $b \Delta$ where $F_{1}, G_{1}$ are in the disc algebra and $G_{1}$ has smooth boundary values. With no loss of generality assume that $G_{1}(0)=0$. By $(3.2) G_{1}=z^{N+1} G_{2}$ where $G_{2}$ is again in the disc algebra and has smooth boundary values so that

$$
P(z) f(z)-F_{1}(z)=\overline{z^{N+1} G_{2}(z)}(z \in b \Delta) .
$$

Suppose for a moment that $G_{2} \not \equiv 0$. We show that there is a constant $\alpha \in \mathbb{C}$ such that $\overline{z^{N+1} G_{2}(z)}+\alpha \neq 0(z \in b \Delta)$ and

$$
\mathcal{W}\left(\overline{z^{N+1} G_{2}(z)}+\alpha\right) \leq-N-1
$$

that is,

$$
\mathcal{W}\left(z^{N+1} G_{2}(z)+\bar{\alpha}\right) \geq N+1 .
$$

The function $\Phi(z)=z^{N+1} G_{2}(z)$ belongs to the disc algebra and has smooth boundary values. It has zero of order at least $N+1$ at the origin. If $\Phi(z) \neq 0(z \in b \Delta)$ then put $\alpha=0$. In this case $\mathcal{W}(\Phi)$ equals the number of zeros of $\Phi$ in $\Delta$ so $\mathcal{W}(\Phi) \geq N+1$. Suppose now that $\Phi(b \Delta)$ contains the origin. Since $\Phi$ has smooth boundary values it follows that $\Phi(b \Delta)$ is nowhere dense. So there are $\alpha$, arbitrarily close to the origin such that $\Phi(z)+\bar{\alpha} \neq 0(z \in b \Delta)$. Let $\nu \geq N+1$ be the multiplicity of the zero of $\Phi$ at the origin. A standard use of the argument principle on a sufficiently small disc $D$ centered at the origin shows that for any $\alpha$ sufficiently close to the origin, $\alpha \neq 0$, the function $z \mapsto \Phi(z)+\bar{\alpha}$ has exactly $\nu$ zeros on $D$ which are arbitrarily close to the origin provided that $\alpha$ is sufficiently close to the origin. Thus, if $\alpha \neq 0$ is sufficiently close to 0 and $\Phi(z)+\bar{\alpha} \neq 0(z \in b \Delta)$ then $\Phi+\bar{\alpha}$ has $\nu$ zeros in a neighbourhood of the origin so the argument principle, now applied to the function $\Phi-\bar{\alpha}$ on $\Delta$, implies that $\mathcal{W}(\Phi+\bar{\alpha}) \geq \nu \geq N+1$ so that (3.3) holds. It follows that $\mathcal{W}\left(P f-F_{1}+\alpha\right) \leq-N-1$. A sufficiently good polynomial approximation $Q$ of $-F_{1}+\alpha$ then satisfies $\mathcal{W}(P f+Q) \leq-N-1$, contradicting (3.1). It follows that $G_{2} \equiv 0$ so $P f=F_{1}$ on $b \Delta$, that is, $P f$ belongs to the disc algebra. We need

PROPOSITION 3.3 [G3, Proposition 5.1, p. 223] Let $\Psi$ be in the disc algebra, let $a \in b \Delta$ and assume that the function $z \mapsto \Psi(z) /(z-a)(z \in b \Delta \backslash\{a\})$ extends continuously to $b \Delta$. Then there is a function $\Psi_{1}$ from the disc algebra such that $\Psi_{1}(z)=\Psi(z) /(z-a)(z \in$ $\bar{\Delta} \backslash\{a\})$.

Proof of Theorem 3.1 continued. Writing $P(z)=p_{0}\left(z-a_{1}\right)\left(z-a_{2}\right) \cdots\left(z-a_{M}\right)$ we have $M \leq N$ and $\left(z-a_{1}\right) \cdots\left(z-a_{M}\right) f(z)=H_{1}(z)(z \in b \Delta)$ where $H_{1}$ belongs to the disc algebra. Let $\alpha_{1}, \cdots, \alpha_{J}$ be those of $a_{1}, \cdots, a_{M}$ which are contained in $\Delta$. By Proposition 3.3 we may write

$$
\left(z-\alpha_{1}\right) \cdots\left(z-\alpha_{J}\right) f(z)=H(z) \quad(z \in b \Delta)
$$

where $H$ belongs to the disc algebra and $J \leq N$. This completes the proof of Theorem 3.1 .

REMARK 3.4 The preceding proof does not work without a smoothness assumption as it is known that there are functions $h$ in the disc algebra such that $h(b \Delta)=h(\bar{\Delta})[\mathrm{G} 1]$.

\section{The general case}


LEMMA 4.1 Let $N \in \mathbb{N}$ and let $f: b \Delta \rightarrow \mathbb{C}$ be a continuous function such that the Fourier series

$$
e^{i(N+1) \theta} f\left(e^{i \theta}\right) \sim \sum_{n=-\infty}^{\infty} A_{n} e^{i n \theta}
$$

of the function $e^{i \theta} \mapsto e^{i(N+1) \theta} f\left(e^{i \theta}\right)$ is such that $A_{0} \neq 0$ and

$$
A_{1}=A_{2}=\cdots=A_{N}=0, \quad A_{-1}=A_{-2}=\cdots=A_{-N}=0 .
$$

There is a polynomial $Q$ such that $f+Q \neq 0$ on $b \Delta$ and

$$
\mathcal{W}(f+Q) \leq-N-1 .
$$

Proof. With no loss of generality we may assume that $A_{0}=1$. Since $z^{N+1} f$ is continuous Fejers theorem implies that $z^{N+1} f$ is the uniform limit of the Cezaro means of its Fourier series [Ho]. So, if

$$
S_{k}\left(e^{i \theta}\right)=\sum_{j=-k}^{k} A_{j} e^{i j \theta} \quad k=0,1,2, \cdots
$$

are the partial sums of the Fourier series then $e^{i(N+1) \theta} f\left(e^{i \theta}\right)$ is the uniform limit, as $m \rightarrow \infty$, of

$$
C_{m}\left(e^{i \theta}\right)=\frac{1}{m+1}\left[S_{0}\left(e^{i \theta}\right)+S_{1}\left(e^{i \theta}\right)+\cdots+S_{m}\left(e^{i \theta}\right)\right]
$$

However, each partial sum $S_{n}$ and therefore each Cezaro mean $C_{m}$ has the same coefficients vanishing property as the one which we have assumed for the Fourier series of $z^{N+1} f$ :

$$
\left(\hat{C_{m}}\right)(0)=1, \quad\left(\hat{C_{m}}\right)(j)=0 \quad(-N \leq j \leq N, j \neq 0)
$$

so that

$$
C_{m}(z)=1+z^{N+1} R_{m}(z)+\overline{z^{N+1} T_{m}(z)}(z \in b \Delta)
$$

where $R_{m}, T_{m}$ are polynomials.

Choose $m$ so large that

$$
\left|C_{m}(z)-z^{N+1} f(z)\right| \leq \frac{1}{2} \quad(z \in b \Delta) .
$$

We have

$$
C_{m}(z)-z^{N+1} R_{m}(z)-z^{N+1} T_{m}(z) \in 1+i \mathbb{R} \quad(z \in b \Delta)
$$

which, by (4.1) implies that

$$
z^{N+1} f(z)-z^{N+1} R_{m}(z)-z^{N+1} T_{m}(z) \in[1 / 2,3 / 2]+i \mathbb{R} \quad(z \in b \Delta) .
$$

It follows that $z^{N+1} f-z^{N+1} R_{m}-z^{N+1} T_{m} \neq 0$ on $b \Delta$ and $\mathcal{W}\left(z^{N+1}\left(f-R_{m}-T_{m}\right)\right)=0$. Thus

$$
\mathcal{W}\left(f-R_{m}-T_{m}\right)=-N-1
$$


so putting $Q=-R_{m}-T_{m}$ completes the proof.

REMARK 4.2 Note that the assumption in Lemma 4.1 is equivalent to saying that $\hat{f}(-N-1) \neq 0$ and $\hat{f}(-1)=\hat{f}(-2)=\cdots=\hat{f}(-N)=0$ and $\hat{f}(-N-2)=\hat{f}(-N-3)=$ $\cdots=\hat{f}(-2 N-1)=0$.

We now turn to the proof of Theorem 1.1. We have already proved the only if part in Section 1. To prove the if part suppose that $f: b \Delta \rightarrow \mathbb{C}$ is a continuous function which satisfies (1.1) for all polynomials $P, Q$ such that $P f+Q \neq 0$ on $b \Delta$. If $N=0$ then we already know that $f$ extends holomorphically through $\Delta$ so assume that $N \geq 1$.

LEMMA 4.3 Let $F: b \Delta \rightarrow \mathbb{C}$ be a continuos function. Assume that for some $N \in \mathbb{N}$ we have

$$
\mathcal{W}(P F+Q) \geq-N
$$

whenever $P, Q$ are polynomials such that $P f+Q \neq 0$ on $b \Delta$. Then

$$
F(z)=G(z)+\overline{H(z)} \quad(z \in b \Delta)
$$

where $G$ belongs to the disc algebra and $H$ is a rational function holomorphic in a neighbourhood of $\bar{\Delta}$.

Assume for a moment that Lemma 4.3 holds. Since our rational function $H$ is smooth on $b \Delta$ the if part of Theorem 1.1 is now an immediate consequence of Lemma 4.3 and Theorem 3.1.

It remains to prove Lemma 4.3. Given an infinite row $A=\left(a_{1}, a_{2}, \cdots\right)$ and $J \in \mathbb{N}$ we denote by $A(J)$ the row containing the first $J$ entries of $A$, that is, $A(J)=\left(a_{1}, a_{2}, \cdots, a_{J}\right)$.

Assume that $F \in C(b \Delta)$ satisfies (4.2) whenever $P, Q$ are polynomials such that $P F+Q \neq 0$ on $b \Delta$. Lemma 4.1 implies that if $P$ is a polynomial such that

$$
\begin{aligned}
& (\hat{P F})(-N-2)=(\hat{P F})(-N-3)=\cdots=(\hat{P F})(-2 N-1)=0 \\
& (\hat{P F})(-1)=(\hat{P F})(-2)=\cdots=(\hat{P F})(-N)=0
\end{aligned}
$$

then $(\hat{P F})(-N-1)=0$. If $P(z)=D_{0}+D_{1} z+\cdots+D_{M} z^{M}$ then

$$
\begin{aligned}
(\hat{P F})(-1) & =D_{0} \hat{F}(-1)+D_{1} \hat{F}(-2)+\cdots+D_{M} \hat{F}(-M-1) \\
& \cdots \\
(\hat{P F})(-N+1) & =D_{0} \hat{F}(-N+1)+D_{1} \hat{F}(-N)+\cdots+D_{M} \hat{F}(-M-N+1) \\
(\hat{P F})(-N) & =D_{0} \hat{F}(-N)+D_{1} \hat{F}(-N-1)+\cdots+D_{M} \hat{F}(-M-N) \\
(\hat{P F})(-N-1) & =D_{0} \hat{F}(-N-1)+D_{1} \hat{F}(-N-2)+\cdots+D_{M} \hat{F}(-M-N-1) \\
(\hat{P F})(-N-2) & =D_{0} \hat{F}(-N-2)+D_{1} \hat{F}(-N-3)+\cdots+D_{M} \hat{F}(-M-N-2) \\
& \cdots \\
(\hat{P F})(-2 N-1) & =D_{0} \hat{F}(-2 N-1)+D_{1} \hat{F}(-2 N-2)+\cdots+D_{M} \hat{F}(-2 N-M-1) .
\end{aligned}
$$


Consider the infinite rows

$$
\begin{aligned}
& X_{-1}=(\hat{F}(-1), \hat{F}(-2), \hat{F}(-3), \cdots) \\
& X_{-2}=(\hat{F}(-2), \hat{F}(-3), \hat{F}(-4), \cdots) \\
& \ldots \\
& X_{-N}=(\hat{F}(-N), \hat{F}(-N-1), \hat{F}(-N-2), \cdots) \\
& X_{-N-1}=(\hat{F}(-N-1), \hat{F}(-N-2), \hat{F}(-N-3), \cdots) \\
& X_{-N-2}=(\hat{F}(-N-2), \hat{F}(-N-3), \hat{F}(-N-4), \cdots) \\
& \ldots \\
& X_{-2 N-1}=(\hat{F}(-2 N-1), \hat{F}(-2 N-2), \hat{F}(-2 N-3), \cdots)
\end{aligned}
$$

The preceding discussion shows that for every $M \in \mathbb{N}$ the following holds: if a row $\left(\overline{D_{0}}, \overline{D_{1}} \cdots \overline{D_{M}}\right)$ is orthogonal to the rows

$$
\begin{array}{r}
X_{-1}(M+1), X_{-2}(M+1), \cdots, X_{-N+1}(M+1), X_{-N}(M+1) \\
X_{-N-2}(M+1), X_{-N-3}(M+1), \cdots, X_{-2 N-1}(M+1)
\end{array}
$$

then it is orthogonal to $X_{-N-1}(M+1)$. This implies that for every $M \in \mathbb{N}$ the row $X_{-N-1}(M+1)$ is a linear combination of $2 M$ rows (4.3). It follows that there are numbers $\lambda_{j},-2 N-1 \leq j \leq-1, j \neq-N-1$, such that

$$
X_{-N-1}=\sum_{-2 N-1 \leq j \leq-1, j \neq-N-1} \lambda_{j} X_{j} .
$$

Consider the function

$$
\Psi(z)=\hat{F}(-N-1) z+\hat{F}(-N-2) z^{2}+\cdots
$$

The function $\Psi$ is holomorphic on $\Delta$ and since

$$
\sum_{n=-\infty}^{-N-1}|\hat{F}(n)|^{2} \leq \sum_{n=-\infty}^{\infty}|\hat{F}(n)|^{2}<\infty
$$

it follows that $\Psi$ belongs to the space $H^{2}[\mathrm{R}]$. We use (4.4) to show that $\Psi$ is a rational function. Note that (4.4) implies that

$$
\begin{aligned}
\Psi(z)=\hat{F}(-N-1) z+\hat{F}(-N-2) z^{2}+\cdots \\
=\lambda_{-1}\left(\hat{F}(-1) z+\hat{F}(-2) z^{2}+\cdots\right)+ \\
+\lambda_{-2}\left(\hat{F}(-2) z+\hat{F}(-3) z^{2}+\cdots\right)+ \\
\quad \ldots \\
+\lambda_{-N}\left(\hat{F}(-N) z+\hat{F}(-N-1) z^{2}+\cdots\right)+ \\
+\lambda_{-N-2}\left(\hat{F}(-N-2) z+\hat{F}(-N-3) z^{2}+\cdots\right)+ \\
\quad \ldots \\
+\lambda_{-2 N-1}\left(\hat{F}(-2 N-1) z+\hat{F}(-2 N-2) z^{2}+\cdots\right)
\end{aligned}
$$


It follows that

$$
\begin{aligned}
\Psi(z) & =\lambda_{-1}\left[P_{-1}(z)+z^{N} \Psi(z)\right]+ \\
& +\lambda_{-2}\left[P_{-2}(z)+z^{N-1} \Psi(z)\right]+ \\
& \cdots \\
& +\lambda_{-N}\left[P_{-N}(z)+z \Psi(z)\right]+ \\
& +\lambda_{-N-2} z^{-1}\left[\Psi(z)-P_{-N-2}(z)\right]+ \\
& +\lambda_{-N-3} z^{-2}\left[\Psi(z)-P_{-N-3}(z)\right]+ \\
& \cdots \\
& +\lambda_{-2 N-1} z^{-N}\left[\Psi(z)-P_{-2 N-1}(z)\right]
\end{aligned}
$$

where

$$
\begin{aligned}
& P_{-1}(z)=\hat{F}(-1) z+\hat{F}(-2) z^{2}+\cdots+\hat{F}(-N) z^{N} \\
& P_{-2}(z)=\hat{F}(-2) z+\hat{F}(-3) z^{2}+\cdots+\hat{F}(-N) z^{N-1} \\
& \ldots \\
& P_{-N}(z)=\hat{F}(-N) z \\
& P_{-N-2}(z)=\hat{F}(-N-1) z \\
& P_{-N-3}(z)=\hat{F}(-N-1) z+\hat{F}(-N-2) z^{2} \\
& { } \\
& P_{-2 N-1}=\hat{F}(-N-1) z+\hat{F}(-N-2) z^{2}+\cdots+\hat{F}(-2 N) z^{N} .
\end{aligned}
$$

So

$$
\left.\begin{array}{c}
\Psi(z)\left[1-\lambda_{-1} z^{N}-\lambda_{-2} z^{N-1}-\cdots-\lambda_{-N} z-\lambda_{-N-2} z^{-1}-\cdots \lambda_{-2 N-1} z^{-N}\right]= \\
=\lambda_{-1} P_{-1}(z)+\lambda_{-2} P_{-2}(z)+\cdots+\lambda_{-N} P_{-N}(z)-\lambda_{-N-2} P_{-N-2}(z) z^{-1}- \\
-\lambda_{-N-3} P_{-N-3}(z) z^{-2}-\cdots-\lambda_{-2 N-1} P_{-2 N-1}(z) z^{-N} .
\end{array}\right\}
$$

Notice that (4.5) implies that there are polynomials $R, S$ with no common factors such that $\Psi(z)=R(z) / S(z)$ where $\mathrm{S}$ has no zero on $\Delta$ since $\Psi$ is holomorphic on $\Delta$. If $\beta_{1}, \cdots \beta_{j}$ are those poles of $\Psi$ that are contained in $b \Delta$ then $\Psi^{*}$, the radial limit function of $\Psi$, satisfies $\Psi^{*}(z)=\Psi(z)\left(z \in b \Delta \backslash\left\{\beta_{1}, \cdots, \beta_{j}\right\}\right)$. However, since $\Psi$ belongs to $H^{2}$ it follows that $\Psi \mid\left(b \Delta \backslash\left\{\beta_{1}, \cdots, \beta_{j}\right\}\right)$ belongs to $L^{2}(b \Delta)[R]$ which is impossible if there is a pole on $b \Delta$ since if $e^{i \tau}$ is such a pole then as $\theta \rightarrow \tau$ the function $\theta \mapsto\left|\Psi\left(e^{i \theta}\right)\right|^{2}$ grows at least as fast as a multiple of $1 /|\theta-\tau|^{2}$ which is not integrable. Thus, $\Psi$ has no poles on $b \Delta$ and consequently $\Psi$ is a rational function holomorphic in a neighbourhood of $\bar{\Delta}$ and so $\Phi(z)=\hat{F}(-1) z+\hat{F}(-2) z^{2}+\cdots=\hat{F}(-1) z+\cdots+\hat{F}(-N) z^{N}+z^{N} \Psi(z)$ is also a rational function holomorphic in a neighbourhood of $\bar{\Delta}$. Thus, $H(z)=\overline{\Phi(\bar{z})}$ is again a rational function holomorphic on a neighbourhood of $\bar{\Delta}$. Note that

$$
\overline{H(z)}=\hat{F}(-1) \frac{1}{z}+\hat{F}(-2) \frac{1}{z^{2}}+\cdots \quad(z \in b \Delta)
$$


Since $H$ is continuous on $b \Delta$ it follows that $G=F-\bar{H}$ is continuous on $b \Delta$ with vanishing Fourier coefficients of negative indices and so $F=G+\bar{H}$ on $b \Delta$ where $G$ is in the disc algebra and $H$ is a rational function holomorphic in a neighbourhood of $\bar{\Delta}$. This completes the proof of Lemma 4.3. The proof of Theorem 1.1 is complete.

\section{Moment conditions and meromorphic extendibility}

Let $f: b \Delta \rightarrow \mathbb{C}$ be a continuous function which extends meromorphically through $\Delta$ and is such that the meromorphic extension has at most $N$ poles in $\Delta$, counting multiplicity. Then there is a nonzero polynomial $P$ of degree not exceeding $N$ such that $P f$ extends holomorphically through $\Delta$. Conversely, if $P$ is a nonzero polynomial of degree not exceeding $N$ such that $P f$ extends holomorphically through $\Delta$ then, after using Proposition 3.3 to factor out the zeros of $P$ on $b \Delta$, we may assume that there are a function $H$ in the disc algebra and a polynomial $Q$ of degree not exceeding $N$ with all zeros contained in $\Delta$, such that $f=H / Q$ on $b \Delta$ which means that $f$ extends meromorphically through $\Delta$ and the meromorphic extension has at most $N$ poles in $\Delta$. Thus, $f$ extends meromorhically through $\Delta$ with at most $N$ poles, counting multiplicity, if and only if there is a nonzero polynomial $P$ of degree not exceeding $N$, such that

$$
(\hat{P f})(-n)=0
$$

for all $n \in \mathbb{N}$. If $P(z)=D_{0}+D_{1} z+D_{2} z^{2}+\cdots+D_{N} z^{N}$ then (5.1) means that

$$
D_{0} \hat{f}(-n)+D_{1} \hat{f}(-n-1)+\cdots+D_{N} \hat{f}(-n-N)=0,
$$

so $f$ extends meromorphically through $\Delta$ if and only if there are complex numbers $D_{0}, \cdots$, $D_{N}$, not all zero, such that (5.2) holds for all $n \in \mathbb{N}$. If this happens then the meromorphic extension of $f$ has at most $N$ poles in $\Delta$, counting multiplicity. Using the reasoning applied in Section 3 we can strenghten this to

PROPOSITION 5.1 Let $f: b \Delta \rightarrow \mathbb{C}$ be a continuous function and let $N \in \mathbb{N}$. Let $D_{0}, D_{1}, \cdots D_{N}$ be a nontrivial solution of the system

$$
\begin{array}{r}
D_{0} \hat{f}(-1)+D_{1} \hat{f}(-2)+\cdots+D_{N} \hat{f}(-1-N)=0 \\
D_{0} \hat{f}(-2)+D_{1} \hat{f}(-3)+\cdots+D_{N} \hat{f}(-2-N)=0 \\
\cdots \\
D_{0} \hat{f}(-N)+D_{1} \hat{f}(-N-1)+\cdots+D_{N} \hat{f}(-2 N)=0
\end{array}
$$

The function $f$ has a meromorphic extension through the unit disc with at most $N$ poles if and only if these numbers $D_{0}, D_{1}, \cdots, D_{N}$ satisfy (5.2) for all $n \in \mathbb{N}, n \geq N+1$.

REMARK 5.2 Note that the system (5.3) is a homogeneous system of $N$ linear equations with $N+1$ unknowns and so it always has a nontrivial solution.

Proof of Proposition 5.1. Observe first that if $a \in \Delta$ and $k \in \mathbb{N}$ then we have

$$
\frac{1}{(z-a)^{k}}=\overline{\frac{z^{k}}{(1-\bar{a} z)^{k}}} \quad(z \in b \Delta)
$$


where the function $z \mapsto z^{k} /(1-\bar{a} z)^{k}$ is holomorphic in a neighbourhood of $\bar{\Delta}$. Note also that if $\Phi$ is in the disc algebra, $m \in \mathbb{N}$ and $a \in \Delta$ then

$$
\frac{\Phi(z)}{(z-a)^{m}}=\frac{\Phi(a)}{(z-a)^{m}}+\frac{\Phi^{\prime}(a)}{(z-a)^{m-1}}+\cdots+\frac{\Phi^{(m-1)}(a)}{(m-1) !(z-a)}+H_{1}(z)
$$

where $H_{1}$ is in the disc algebra. Using decomposition into partial fractions we now see that whenever $g$ is of the form

$$
g(z)=\frac{\Phi(z)}{\left(z-a_{1}\right)^{k_{1}}\left(z-a_{2}\right)^{k_{2}} \cdots\left(z-a_{J}\right)^{k_{J}}}
$$

with $\Phi$ in the disc algebra and $a_{j} \in \Delta(1 \leq j \leq J)$ then

$$
g(z)=F(z)+\overline{G(z)} \quad(z \in b \Delta)
$$

where $F$ is in the disc algebra and $G$ is a rational function holomorphic in a neighbourhood of $\bar{\Delta}$.

If $D_{0}, D_{1}, \cdots D_{N}$, not all of them being zero, satisfy (5.3) and (5.2) for all $n \geq N+1$, then they satisfy (5.2) for all $n \in N$ so by the preceding discussion $f$ extends meromorphically through $\Delta$ and the meromorphic extension has at most $N$ poles, counting multiplicity.

To prove the converse, assume that there are numbers $a_{1}, a_{2}, \cdots, a_{J}$ in $\Delta$, positive integers $k_{1}, k_{2}, \cdots, k_{J}$ such that $k_{1}+k_{2}+\cdots+k_{J} \leq N$, and a function $\mathrm{H}$ from the disc algebra such that

$$
f(z)=\frac{H(z)}{\left(z-a_{1}\right)^{k_{1}} \cdots\left(z-a_{J}\right)^{k_{J}}} \quad(z \in b \Delta) .
$$

By the argument principle it follows that

$$
\left.\begin{array}{rl}
\mathcal{W}(P f+Q) \geq-N & \text { whenever } P, Q, \text { are } \\
\text { polynomials such that } & P f+Q \neq 0 \text { on } b \Delta .
\end{array}\right\}
$$

Let $P(z)=D_{0}+D_{1} z+\cdots+D_{N} z^{N}$ be a nonzero polynomial such that $(\hat{P} f)(j)=0 \quad(-N \leq$ $j \leq-1)$, that is, let $D_{0}, D_{1}, \cdots D_{N}$, not all being zero, satisfy (5.3). By the preceding discussion

$$
(P f)(z)=F(z)+\overline{z^{N+1} G(z)}(z \in b \Delta)
$$

where $\mathrm{F}$ is in the disc algebra and $G$ is a rational function holomorphic in a neighbourhood of $\bar{\Delta}$. In particular, $G$ is smooth on $b \Delta$. Assume for a moment that $G \not \equiv 0$. Then, as in the proof of Theorem 3.1, we find an $\alpha \in \mathbb{C}$ such that $P f-F-\alpha \neq 0$ on $b \Delta$ and that $\mathcal{W}(P f-F-\alpha) \leq-N-1$. A sufficiently good polynomial approximation $Q$ of $-F-\alpha$ then satisfies $\mathcal{W}(P f+Q) \leq-N-1$ which contradicts (5.4). It follows that $G \equiv 0$ so $P f=F$ where $F$ is in the disc algebra and consequently $(\hat{P f})(j)=0(j \leq-N-1)$, that is, (5.2) is satisfied for all $n, n \geq N+1$. This completes the proof. 


\section{Remarks}

Theorem 1.1 is a one-variable theorem about meromorphic extensions of continuous functions on the unit circle. It can be described also in more geometric terms as a theorem in $\mathbb{C} \times \overline{\mathbb{C}}$ as follows. Let $f: b \Delta \rightarrow \mathbb{C}$ be a continuous function. Then its graph $\Gamma_{f}=$ $\{(z, f(z)): z \in b \Delta\}$ is a simple closed curve. Suppose that $P, Q$ are polynomials such that $P f+Q \neq 0$ on $b \Delta$, that is, such that the variety

$$
V=\left\{(z, w) \in \mathbb{C}^{2}: P(z) w+Q(z)=0\right\}
$$

misses $\Gamma_{f}$. In the special case when $f$ is smooth then the graf $\Gamma_{f}$ is a smooth curve and the linking number $\operatorname{link}\left(\Gamma_{f}, V\right)$ is well defined $[\mathrm{AW}]$ and is equal to $\mathcal{W}(P f+Q)$ [AW, Lemma 1.2, p.130]. If $f$ is merely continuous then for all smooth curves $\Gamma$ homotopic to $\Gamma_{f}$ in $\mathbb{C}^{2} \backslash V$ the linking number $\operatorname{link}(\Gamma, V)$ is the same which implies that for a continuous function $f$ such that $\Gamma_{f}$ misses $V$ we may define $\operatorname{link}\left(\Gamma_{f}, V\right) \operatorname{simply}$ as $\operatorname{link}\left(\Gamma_{g}, V\right)$ where $\Gamma_{g}=\{(z, g(z)): z \in b \Delta\}$ is the graph of a sufficiently good smooth approximation $g: b \Delta \rightarrow$ of $f$, so we have $\operatorname{link}\left(\Gamma_{f}, V\right)=\mathcal{W}(P g+Q)=\mathcal{W}(P f+Q)$.

If $f$ has a meromorphic extension $\tilde{f}$ through $\Delta$ then the graph $\{(z, \tilde{f}(z)): z \in \Delta\}$ of $\tilde{f}$ is a complex submanifold of $\Delta \times \overline{\mathbb{C}}$ attached to $b \Delta \times \overline{\mathbb{C}}$ along $\Gamma_{f}$. So Theorem 1.1 says that the curve $\Gamma_{f}$ bounds a submanifold of $\Delta \times \overline{\mathbb{C}}$ (that is a graph over $\Delta$ ) if and only if the linking numbers $\operatorname{link}\left(\Gamma_{f}, V\right)$ for algebraic varieties $V$ of the form (1.2) which miss $\Gamma_{f}$, are bounded from below. Since $f$ is only assumed to be continuous, our curve $\Gamma_{f}$ is not smooth, although, being a graph over $b \Delta$ it is quite special. For general curves $\Gamma$ there are recent results in a similar spirit, under the assumption of real analyticity and with the assumption on linking numbers made for all algebraic varieties which miss $\Gamma$ [HL, Th.6.6].

In Theorem 3.1 it is enough to assume (1.1) only for polynomials $P$ of degree not exceeding $N$. We are not able to see that the same holds for Theorem 1.1. In our proof of Theorem 1.1 we need polynomials of arbitrarily high degree to prove that (1.1) implies that

$$
f(z)=G(z)+\overline{H(z)} \quad(z \in b \Delta)
$$

where $H$ is a rational function holomorphic in a neighbourhood of $\bar{\Delta}$. We can then use only the smoothness of $H$ on $b \Delta$ to be able to apply Theorem 1.1 to show that the meromorphic extension of $f$ has at most $N$ poles in $\Delta$. On the other hand, once we know that $f$ is of the form (6.1) where $H$ is a rational function holomorphic in a neighbourhood of $\bar{\Delta}$ then we can, alternatively, show directly that the number of poles in $\Delta$ does not exceed $N$ by using

LEMMA 6.1 Let $\Psi$ be in the disc algebra and let $a_{1}, a_{2} \cdots a_{m} \in \Delta$ be such that $\Psi\left(a_{j}\right) \neq$ $0(1 \leq j \leq m)$. There is a polynomial $Q$ such that $\Psi+\left(z-a_{1}\right) \cdots\left(z-a_{m}\right) Q$ has no zero on $\bar{\Delta}$.

Indeed, assuming Lemma 6.1 for a moment, one observes that if $f$ is of the form (6.1)where $H$ is a rational function holomorphic in a neighbourhood of $\bar{\Delta}$ then $f$ must be of the form

$$
f(z)=\frac{\Psi(z)}{\left(z-a_{1}\right) \cdots\left(z-a_{m}\right)} \quad(z \in b \Delta)
$$


where $\Psi$ is in the disc algebra, $a_{j} \in \Delta(1 \leq j \leq m)$ and $\Psi\left(a_{j}\right) \neq 0(1 \leq j \leq m)$. Then one uses Lemma 6.1 and (1.1) to show that

$$
\mathcal{W}(f+Q)=\mathcal{W}\left(\frac{\Psi+\left(z-a_{1}\right) \cdots\left(z-a_{m}\right) Q}{\left(z-a_{1}\right) \cdots\left(z-a_{m}\right)}\right)=-m \geq-N
$$

so that $m \leq N$ what we wanted to show.

Proof of Lemma 6.1. Let $c_{0}, c_{1}, \cdots c_{m} \in \mathbb{C}, c_{0} \neq 0$. Computing higher order derivatives of $z \mapsto e^{\Phi(z)}$ it is easy to see that there are numbers $d_{0}, d_{1}, \cdots, d_{m} \in \mathbb{C}$ such that if $\Phi$ is holomorphic in a neighbourhood of 0 with Taylor expansion

$$
\Phi(z)=d_{0}+d_{1} z+\cdots d_{m} z^{m}+\cdots
$$

then $e^{\Phi}$ has Taylor expansion

$$
e^{\Phi(z)}=c_{0}+c_{1} z+c_{2} z^{2}+\cdots c_{m} z^{m}+\cdots .
$$

Note that it is enough to construct a $Q$ in the disc algebra as then a sufficiently good polynomial approximation of $Q$ will have all the required properties.

Write $\left(z-a_{1}\right) \cdots\left(z-a_{m}\right)=\left(z-\alpha_{1}\right)^{p_{1}} \cdots\left(z-\alpha_{k}\right)^{p_{k}}$ where $p_{1}+\cdots+p_{k}=m$ and where $\alpha_{i} \neq \alpha_{j}(i \neq j)$. Our $Q$ will have to satisfy $\Psi+\left(z-\alpha_{1}\right)^{p_{1}} \cdots\left(z-\alpha_{k}\right)^{p_{k}}=e^{\Phi}$ with $\Phi$ from the disc algebra which means that for each $j, 1 \leq j \leq k$, the function $e^{\Phi}-H$ must have zero of order at least $p_{j}$ at the point $\alpha_{j}$. This means that for each $j, 1 \leq j \leq k$, the Taylor expansion of $e^{\Phi}$ in a neighbourhood of $\alpha_{j}$ has the form

$$
e^{\Phi(z)}=\Psi\left(\alpha_{j}\right)+\cdots+\frac{\Psi^{\left(p_{j}\right)}\left(\alpha_{j}\right)}{p_{j} !}\left(z-\alpha_{j}\right)^{p_{j}}+\cdots
$$

By the preceding discussion there are numbers $d_{j, \ell}, 0 \leq \ell \leq p_{j}, 1 \leq j \leq k$, such that if for each $j, 1 \leq j \leq k$ the function $\Phi$ satisfies

$$
\Phi(z)=d_{j, 0}+d_{j, 1}\left(z-\alpha_{j}\right)+\cdots+d_{j, p_{j}}\left(z-\alpha_{j}\right)^{p_{j}}+\cdots
$$

then (6.2) holds for each $j, 1 \leq j \leq k$. It is an easy application of the Weierstrass factorization theorem to construct an entire function $\Phi$ with this property $[R$, Th. 15.13, p. 304]. The function

$$
Q=\frac{e^{\Phi}-\Psi}{\left(z-\alpha_{1}\right)^{p_{1}} \cdots\left(z-\alpha_{k}\right)^{p_{k}}}
$$

will have the required properties. This completes the proof.

The following question is open:

QUESTION 6.2 Let $f: b \Delta \rightarrow \mathbb{C}$ be a continuous function. Suppose that for some $N \in \mathbb{N}$ we have $\mathcal{W}(f+Q) \geq-N$ for all polynomials $Q$ such that $f+Q \neq 0$ on $b \Delta$. Must $f$ extend meromorphically through $\Delta$ ? 
In other words, we are asking whether in Theorem 1.1 it is enough to assume that $P \equiv$ 1 or, equivalently, whether the precise analogue of Theorem 1.0 holds for meromorphic extendibility. We do not know the answer even in the case when $f$ is smooth.

We conclude with a remark about holomorphic extendibility. It is an obvious question whether Theorem 1.0 holds for a smaller class of polynomials $Q$. That linear polynomials do not suffice was shown in [W], that polynomials of uniformly bounded degree do not suffice was shown in [G2]. One may ask, for instance, whether the polynomials $Q$ satisfying $Q(0)=0$ suffice. The answer is no as shown by the example $f(z)=z /(z-1 / 2)(z \in b \Delta)$. Indeed, writing $Q(z)=z Q_{1}(z)$ where $Q_{1}$ is a polynomial the argument principle implies that

$$
\mathcal{W}(f+Q)=\mathcal{W}\left(\frac{z}{z-1 / 2}+Q\right)=\mathcal{W}\left(\frac{z\left[1+(z-1 / 2) Q_{1}\right]}{z-1 / 2}\right) \geq 0
$$

for all polynomials $Q$ such that $Q(0)=0$ and such that $f+Q \neq 0$ on $b \Delta$, yet $f$ does not extend holomorphically through $\Delta$. However, there is no such example if $f$ has a meromorphic extension through $\Delta$ which does not vanish at 0 by the following

PROPOSITION 6.3 Let $S$ be a polynomial with all its zeros contained in $\Delta$. Suppose that $f: b \Delta \rightarrow \mathbb{C}$ is a continuous function such that $\mathcal{W}(f+S Q) \geq 0$ for each polynomial $Q$ such that $f+S Q \neq 0$ on $b \Delta$. If $f$ extends meromorphically through $\Delta$ and the meromorphic extension has no common zeros with $S$ then $f$ extends holomorphically through $\Delta$.

Proof. Suppose that

$$
f(z)=\frac{H(z)}{\left(z-a_{1}\right) \cdots\left(z-a_{N}\right)} \quad(z \in b \Delta)
$$

where $a_{j} \in \Delta(1 \leq j \leq N)$ and where $H$ is in the disc algebra, $H\left(a_{j}\right) \neq 0(1 \leq j \leq N)$, such that $H$ has no common zero with $S$. By Lemma 6.1 there is a polynomial $Q$ such that $H+\left(z-a_{1}\right) \cdots\left(z-a_{N}\right) S Q$ has no zero on $\bar{\Delta}$ It follows that

$$
0 \leq \mathcal{W}(f+S Q)=\mathcal{W}\left(\frac{H+\left(z-a_{1}\right) \cdots\left(z-a_{N}\right) S Q}{\left(z-a_{1}\right) \cdots\left(z-a_{N}\right)}\right)=-N
$$

which implies that $N=0$, so $f$ extends holomorphically through $\Delta$. This completes the proof.

ACKNOWLEDGEMENTS The author is grateful to Sergey Ivashkovich for explaining the simple facts about meromorphic extendibility mentioned in the beginning of Section 5 .

This work was supported in part by the Ministry of Higher Education, Science and Technology of Slovenia through the research program Analysis and Geometry, Contract No. P1-0291 


\section{REFERENCES}

[AW] H. Alexander and J. Wermer: Linking numbers and boundaries of varieties. Ann. Math. 151 (2000) 125-150

[G1] J. Globevnik: The range of analytic extensions.

Pacif. J. Math. 69 (1977) 365-384

[G2] J. Globevnik: Holomorphic extendibility and the argument principle.

Complex Analysis and Dynamical Systems II. (Proceedings of a conference held in honor of Professor Lawrence Zalcman's sixtieth birthday in Nahariya, Israel, June 9-12, 2003), Contemp. Math. 382 (2005) 171-175

[G3] J. Globevnik: The argument principle and holomorphic extendibility to finite Riemann surfaces.

Math. Z. 253 (2006) 219-225

[HL] F. R. Harvey and H. B. Lawson, Jr.: Projective linking and boundaries of positive holomorphic chains in projective manifolds, Part I.

Preprint, http://www.arxiv.org/abs/math.CV/0512379

[Ho] K. Hoffman: Banach Spaces of Analytic Functions.

Prentice Hall, Englewood Cliffs, 1962

[R] W. Rudin: Real and Complex Analysis.

McGraw-Hill, New York, 1987

[S] E. L. Stout: Boundary values and mapping degree.

Michig. Math. J. 47 (2000) 353-368

[W] J. Wermer: The argument principle and boundaries of analytic varieties.

Oper. Theory Adv. Appl., 127, Birkhauser, Basel, 2001, 639-659

[Z] A. Zygmund: Trigonometric series.

Cambridge University Press, Cambridge, New York, 1959

Institute of Mathematics, Physics and Mechanics

University of Ljubljana, Ljubljana, Slovenia

josip.globevnik@fmf.uni-lj.si 